\title{
Discovery of a histidine-based scaffold as an inhibitor of gut microbial choline trimethylamine-lyase
}

\author{
Moustafa Gabr, ${ }^{*[a] \#}$ and Katarzyna Świderek ${ }^{*[b] \#}$ \\ [a] Dr M. Gabr \\ Department of Radiology \\ Stanford University School of Medicine \\ Stanford, CA 94305, USA \\ E-mail: gabr@stanford.edu \\ [b] Dr K. Świderek \\ Departament de Química Física i Analítica \\ Universitat Jaume I \\ 12071 Castellón, Spain \\ E-mail: swiderek@uji.es \\ \#These authors contributed equally to this work.
}

Supporting information for this article is given via a link at the end of the document
Subsequently, TMA is oxidized to TMAO by flavin-dependent monooxygenase 3 (FMO3) in human liver (Figure 1a). ${ }^{[16]}$ These metabolic events in gut microbiota and liver are identified as potential therapeutic targets for cardiovascular diseases and CKD in order to inhibit the production of TMAO.$^{[17,18]}$ For example, targeting hepatic $\mathrm{FMO} 3$ with antisense oligonucleotides reduced TMAO levels as well as atherosclerosis in mice models. ${ }^{[19]}$ Accumulation of TMA and hepatic inflammation are common side effects for blocking FMO3 activity, thus, targeting TMA-lyase has evolved as a more promising approach to reduce TMAO levels. ${ }^{[19-}$ 21]

In vitro characterization of the catalytic activity of CutC and substrate specificity using homology modeling and mutagenesis experiments enabled research efforts to develop CutC inhibitors. ${ }^{[22]}$ The choline utilization (cut) gene cluster, identified in human gut isolates, encodes the glycyl radical enzyme CutC as well as its activating protein, CutD, and mediates anaerobic metabolism of choline. ${ }^{[15,22,23]}$ It is proposed that CutC functions via a glycyl radical mechanism that starts with hydrogen abstraction from a cysteine residue (Cys489) producing a thienyl radical intermediate which triggers the interaction with choline. ${ }^{[15,22]}$

The first CutC inhibitor (1 in Figure 1b), 3,3-dimethyl-1-butanol (DMB), was designed as a structural analogue of choline by replacing its nitrogen atom with a carbon atom. ${ }^{[17]}$ Non-lethal inhibition of TMA production was exerted by DMB in cultured microbes. Moreover, oral administration of DMB reduced TMAO levels in mice with access to a high choline diet. ${ }^{[17]}$ However, evaluation of the CutC inhibitory activity of DMB in vitro and in bacterial cell culture revealed poor CutC inhibitory profile. ${ }^{[24]}$ These findings suggests that in vivo reduction in TMA production might be attributed to the interaction of DMB with alternative targets. Hazen and coworkers further reported iodomethylcholine (IMC, 2 in Figure 1b) as a choline analogue with a demonstrated ability to reduce TMAO production in vivo. ${ }^{[18]}$ Recently, betaine aldehyde ( $\mathbf{3}$ in Figure $1 \mathrm{~b}$ ) has been reported as CutC inhibitor with weak to moderate inhibitory activity (half maximal inhibitory concentration $\left(\mathrm{IC}_{50}\right)$ value of $26 \mu \mathrm{M} \cdot{ }^{[24]} \mathrm{A}$ cocrystal structure of 3 with CutC (PDB ID: 5FAU) revealed that the interaction is mediated through a covalent thiohemiacetal linkage. ${ }^{[24]}$ A cyclic analogue of choline (4 in Figure 1b) has displayed $\sim 10$-folds 
improvement in in vitro CutC inhibitory activity in comparison to 3. ${ }^{[25]}$ However, the evaluation of the ability of $\mathbf{4}$ to inhibit the conversion of choline to TMA ex vivo revealed half maximal effective concentration $\left(E_{50}\right)$ value of $60 \mu \mathrm{M}^{\left[{ }^{[25]}\right.}$ These findings limits the potential of $\mathbf{4}$ as a lead for further structural optimization to develop CutC inhibitors. Unlike therapeutics with established ability to manipulate gut microbiota (e.g. antibiotics and prebiotics), small molecules hold promise as potential gut microbiota-targeted therapeutics with limited alteration to multiple gut microbial activities. ${ }^{[24]}$ Thus, there is an unmet need to identify leads as small-molecule CutC inhibitors that possess in vitro and ex vivo CutC inhibitory activity.

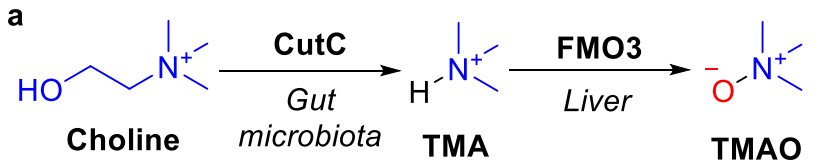<smiles>CC(C)(C)CCO</smiles><smiles>C[N+](C)(CI)CCO</smiles><smiles>C[N+](C)(C)CC=O</smiles>

3 activity of compounds $\mathbf{5 - 1 2}$ identified compound $\mathbf{5}$ (Figure 3 ) as an inhibitor of CutC.

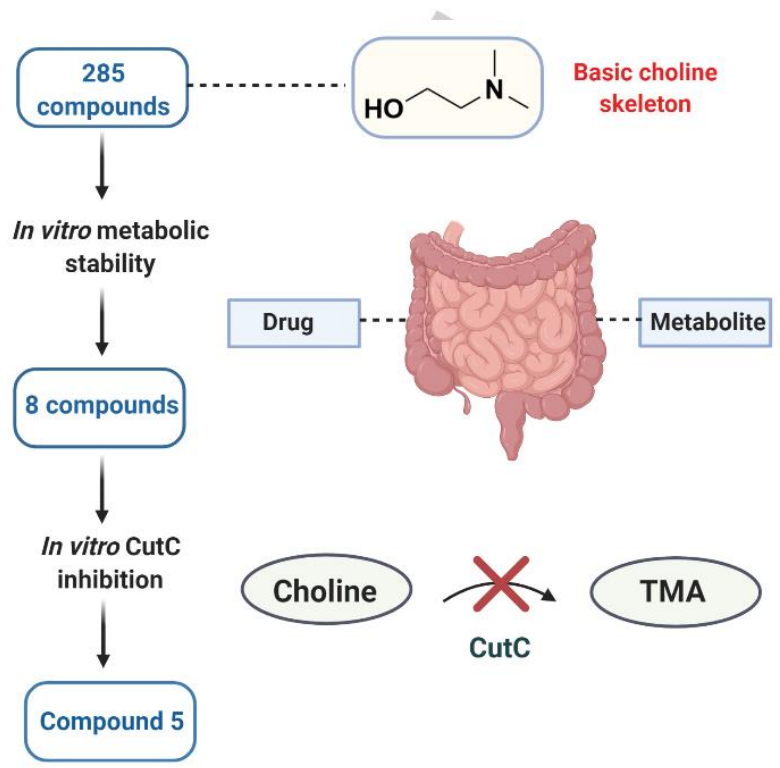

Figure 2. A schematic illustration of the workflow used in this study to identify CutC inhibitors with in vitro intestinal metabolic stability.

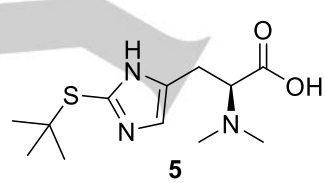<smiles>CC(C)CC1(O)CCCC1N(C)C</smiles><smiles>[3H]c1ccccc1CC(C(=O)O)(C(=O)O)N(C)C</smiles>

Chemical structures of CutC inhibitors.

The impact of gut microbiota on the metabolic stability of drugs has been recognized as a critical factor controlling their therapeutic outcome. ${ }^{[26,27]}$ Intestinal metabolism significantly contributes to drug bioavailability and pharmacokinetics. ${ }^{[28,29]}$ Therefore, intestinal metabolic stability of leads as gut microbiotatargeted small molecules is a key requirement for their in vivo efficacy and clinical translation. Herein, we develop a workflow for the identification of competitive inhibitors of CutC that demonstrate intestinal metabolic stability.

The workflow adapted in this study is shown in Figure 2. A small focused library ( 285 compounds) of commercially available small molecules that maintain the basic chemical skeleton of a neutral analog of choline was established. The recent superior CutC inhibitory activity of a cyclic and neutral choline analogue $\mathbf{4}$ over previously charged choline analogues 2 and $\mathbf{3}$ has directed our efforts to this scaffold for selection of compounds. ${ }^{[25]}$ The focused library was subjected to in vitro metabolic screening using mixed gender human intestinal S9 fraction. The compounds were tested at a final concentration of $33 \mu \mathrm{M}$ which is in agreement with the estimated drug concentration in human gastrointestinal tract. ${ }^{[30]}$ The percentage of intact parent compound upon incubation with intestinal S9 fraction was determined using Liquid Chromatography-Mass Spectrometry (LC-MS) analysis. Eight compounds $\mathbf{5 - 1 2}$ (Figure 3 ) indicated $\sim 90 \%$ intestinal metabolic stability (i.e. $90 \%$ intact compound) as revealed by LC-MS analysis after 2 hours incubation (Table S1, Supporting Information). Additional screening for CutC in vitro inhibitory<smiles>CCCCI(C)C(C)CC</smiles><smiles>CCC(C)(C(O)c1cncnc1)N(C)C</smiles><smiles>CN(C)C(c1ccccc1)C1(O)CCCC1</smiles>

Figure 3. Chemical structures of compounds 5-12.

Compounds 5-12 were initially screened for their ability to inhibit anaerobic choline metabolism by the cut gene clustercontaining human gut isolate Escherichia coli (E. coli) MS 200-1 using compound $\mathbf{4}$ as a positive control. The screening was performed in a five-dose format for each tested compound by supplementing the bacterial culture with $d g$-choline as a substrate for the investigated metabolic pathway. Production of $d_{g}$-TMA was next assessed using Liquid Chromatography-Mass Spectrometry (LC-MS). As shown in Table 1, compound 5 exhibited an EC $_{50}$ value of $4.3 \pm 0.9 \mu \mathrm{M}$ in the whole cell assay (in comparison to $\mathrm{EC}_{50}$ value of $12+1.8 \mu \mathrm{M}$ for 4$)$. Uptake studies using $E$. coli MS 200-1 (Figure S1, Supporting Information) revealed no impact of 
compound $\mathbf{5}$ on the bacterial uptake of different choline concentrations $(0-200 \mu \mathrm{M})$. Thus, direct interference of compound $\mathbf{5}$ with choline-metabolizing enzymes rather than altering choline uptake is likely to mediate the inhibitory effect of compound $\mathbf{5}$ on TMA production. Compounds $\mathbf{6}$ and $\mathbf{7}$ exhibited weak to moderate inhibitory activity on anerobic choline metabolism. Additionally, compounds 8-12 poorly inhibited choline metabolism in the whole cell assay (Table 1).

Table 1. Evaluation of the activity of compounds 5-12 in whole cell assay and in vitro CutC assay.

\begin{tabular}{ccc}
\hline Compound & $\begin{array}{c}\text { EC } 50 \text { against } \\
\text { E. coli MS 200-1 } \\
(\mu \mathrm{M})\end{array}$ & $\begin{array}{c}\text { IC } 5 \text { against } \\
\text { CutC }(\mu \mathrm{M})\end{array}$ \\
\hline $\mathbf{4}$ & $12+1.8$ & $4.1+0.3$ \\
$\mathbf{5}$ & $4.3 \pm 0.9$ & $1.9 \pm 0.2$ \\
6 & $180+15$ & $79+4.7$ \\
7 & $280+39$ & $>500$ \\
8 & $>1000$ & $>500$ \\
9 & $>1000$ & $>500$ \\
10 & $>1000$ & $>500$ \\
11 & $>1000$ & $>500$ \\
12 & $>1000$ & \\
\hline
\end{tabular}

In vitro CutC inhibitory assay was performed for compound $\mathbf{5}$ in order to verify that reduction in TMA production by 5 in the whole cell assay is associated to direct CutC inhibition. Wild-type CutC from Desulfovibrio desulfuricans strain G20 was expressed and purified as previously described. ${ }^{[22]}$ The in vitro assay is based on coupling the activity of CutC to the reduction of acetaldehyde by $\mathrm{NADH}$-dependent yeast alcohol dehydrogenase (YADH). Dosedependent screening of compound $\mathbf{5}$ revealed an $\mathrm{IC}_{50}$ value of $1.9 \pm 0.2 \mu \mathrm{M}$ (Table 1 and Figure 4 ) in comparison to $\mathrm{IC}_{50}$ value of $4.1+0.3 \mu \mathrm{M}$ for 4 . Therefore, inhibition of CutC is demonstrated as working mechanism for the ability of compound $\mathbf{5}$ to reduce TMA production in whole cell assay. Additionally, compound 6 featured weak in vitro inhibition of CutC with $\mathrm{IC}_{50}$ value of $79+4.7$ $\mu \mathrm{M}$. In consistence with whole cell assays, compounds 8-12 didn't inhibit CutC activity in vitro.

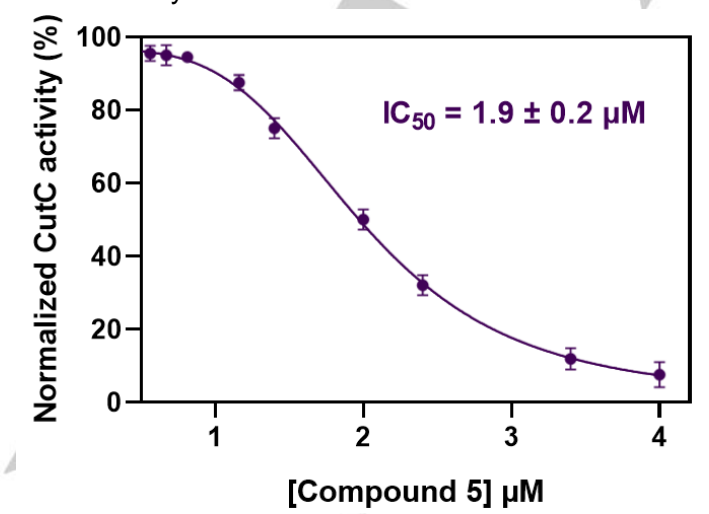

To further investigate CutC inhibitory profile of compound $\mathbf{5}$, a kinetic study for CutC activity was performed. Various concentrations of choline, CutC substrate, were subjected to the enzymatic reaction catalyzed by CutC in the presence and absence ofcompound $\mathbf{5}$. Michaelis-Menten plot of the reaction velocity against substrate concentration revealed that $V_{\max }$, maximum rate for enzymatic reaction, was unchanged in the absence and the presence of $6 \mu \mathrm{M}$ of compound 5 (Figure 5). However, Michaelis constant $\left(\mathrm{K}_{\mathrm{M}}\right)$ remarkably increased from 395 $\mu \mathrm{M}$ to $1626 \mu \mathrm{M}$ in the presence of $6 \mu \mathrm{M}$ ofcompound 5 . These findings are in consistence with the profiles of competitive inhibitors of enzyme activities according to Michaelis-Menten equation. ${ }^{[31]}$ Therefore, we propose that compound $\mathbf{5}$ functions as a competitive inhibitor of CutC in the biochemical screening and whole cell assay.

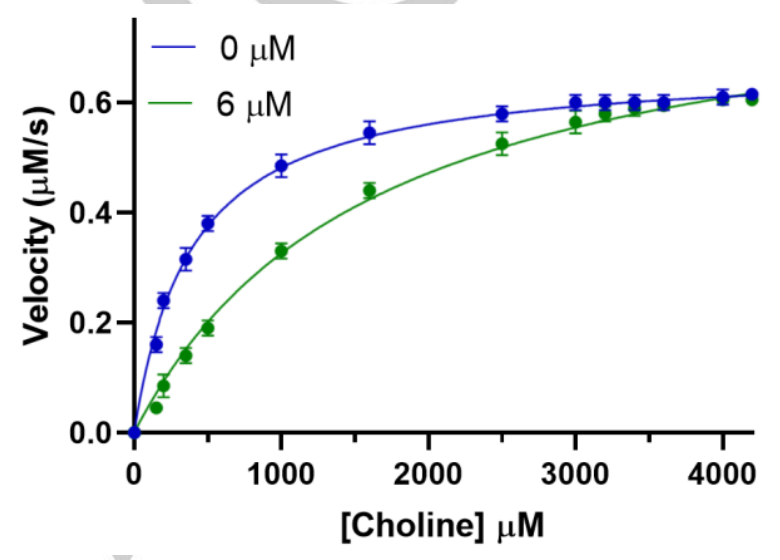

Figure 5. Michaelis-Menten plot of the reaction velocity against various concentrations of choline in the absence and presence of compound $5(6 \mu \mathrm{M})$ Error bars represent standard deviation $(n=3)$.

Based on the kinetic results as a competitive inhibitor, compound $\mathbf{5}$ is expected to bind in the same pocket as choline ion Nevertheless, the pattern of interactions for compound $\mathbf{5}$ within the active site is not so evident. As shown in Figure 6, substrate and inhibitor are significantly different, not only due to their size but importantly because of the charge distribution. Fragment of compound $\mathbf{5}$, that mimics to some extent the structure of choline, is modified by introduction of a carboxyl group in the place of alcohol hence generating a strong $\mathrm{H}$-bond acceptor. Moreover, one of the three methyl groups originally attached to ethanolamine is substituted by a hydrogen atom. Removal of steric hindrance by the introduction of hydrogen atom ensures better access to the positively charged nitrogen. Generally, four possible acceptors and two donors of hydrogen bond can be identified in compound 5 in its zwiterrionic form. Modified ethanolamine group, similar as in choline ion, can create attractive interactions with negatively charged moieties. However, in compound 5, this group can additionally serve as a hydrogen bond donor, unlike in the substrate.

Figure 4. Dose-response curves of compound $\mathbf{5}$ in CutC in vitro inhibition assay. Error bars represent standard deviation $(n=3)$. 


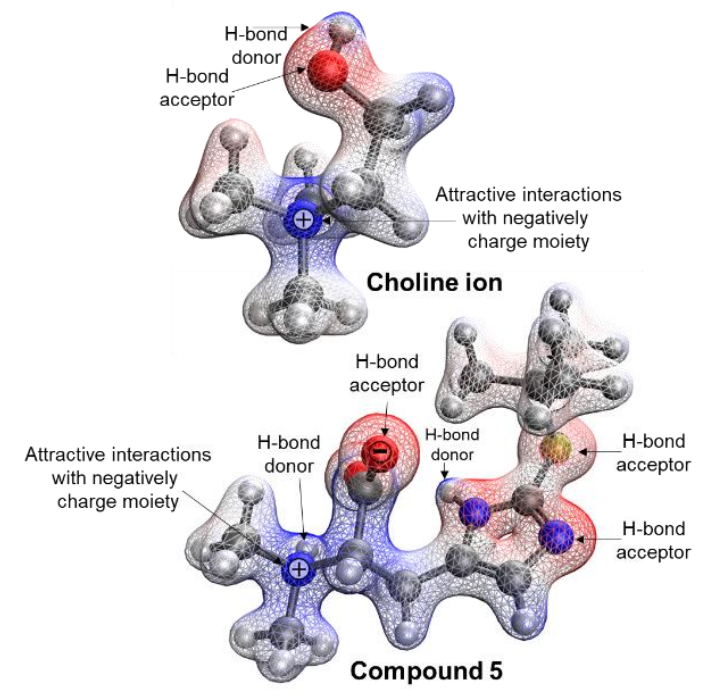

Figure 6. Map of electrostatic potential generated by choline ion (on the top) and zwitterionic form of compound $\mathbf{5}$ (on the bottom) obtained based on the distribution of ChelpG ${ }^{[32]}$ charges computed for optimized structures of substrate and inhibitor at M06-2X[33,34]/6-31+(d,p) level of theory in continuum water model $(\mathrm{CPCM})^{[35-38]}$ using Gaussian 09. ${ }^{[39]}$

Therefore, it is expected that compound $\mathbf{5}$ creates a pattern of interactions with the active site different from the substrate. To identify the interactions between the proposed inhibitor and CutC, classical molecular dynamic (MD) simulations were employed (see Supporting Information for details). Two different conformers of CutC were used in computational studies. The first one (closedCutC) represented by the crystallographic structure of the protein from Desulfovibrio alaskensis G20 (PDB ID 5fau), ${ }^{[23]}$ corresponds to the conformer with the active site pocket completely closed and perfectly fitted to the bound choline ion. The second structure (open-CutC) was obtained by homology modelling done using SwissMode ${ }^{[40-44]}$ with 'choline-free form' of CutC from Klebsiella pneumoniae as a template (PDB ID 5a0z). ${ }^{[45]}$ This substrate-free form of the protein is believed to possess a tunnel to the outside that could allow for the access of large molecules to the active site. Therefore, this structure was used to build the model of the protein in complex with an inhibitor. Indeed, the entrance to the binding tunnel was found to be located between two $\alpha$-helices formed by $831-839$ and $703-708$ residues. The entrance gate is almost double size in the open-(11.2 $\AA$ ) in comparison to the closed-CutC $(6.5 \AA)$ conformation. Analysis of the structures revealed that open and closed conformation can be achieved due to the presence of the highly flexible backbone of C-terminus, as shown in Supporting Information.

The putative interactions between compound $\mathbf{5}$ and the active site of CutC were determined based on the results obtained from unbiased MD simulations and, subsequently, they were compared with those established for choline ion. Protein models in complex with choline ion and compound $\mathbf{5}$ were neutralized and placed in a box of water molecules. Then the system was equilibrated at $37^{\circ} \mathrm{C}$ employing non-accelerated classical NVT $\mathrm{MD}$ with $\mathrm{AMBER}{ }^{[46]}$ force field as implemented in NAMD ${ }^{[47]}$ package (see Supporting Information). The validation of the docked pose predicted for compound $\mathbf{5}$ was done by employing five independent $M D$ simulations of $10 \mathrm{~ns}$ each at $37^{\circ} \mathrm{C}$, starting from different structures generated during equilibration process in different temperatures $\left(27,32,37,42\right.$ and $\left.47^{\circ} \mathrm{C}\right)$. The key interactions of the substrate and inhibitor with the active site delivered from these studies are shown in Figure 7.

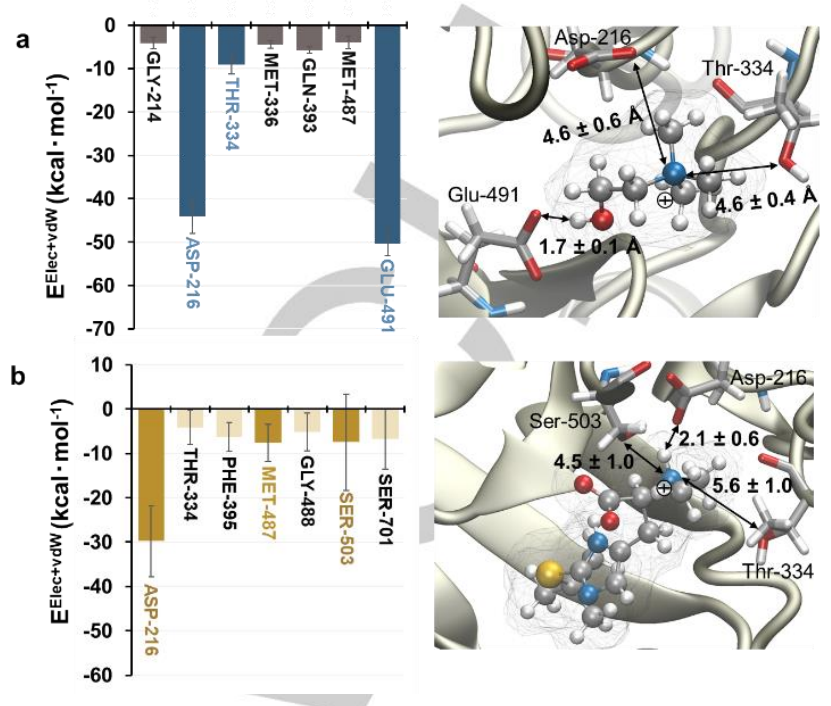

Figure 7. Averaged favorable interaction energies $\left(E^{E l e c+v d W}\right)$ (electrostatic and Lennard-Jones) for a. choline ion identified over 1000 structures from one trajectory and $\mathbf{b}$. compound $\mathbf{5}$ identified over $\mathbf{5 0 0 0}$ structures delivered from five independent non-accelerated classical MD simulations, respectively together with the representative structures of the CutC active site with bound substrate and inhibitor.

Compound 5 was found to create several favorable interactions with residues located in the interior of the active site. Despite the loss of electrostatic contact with Glu491 dictated by the presence of the negatively charged carboxylic group in the inhibitor structure, interactions with the remaining two key residues for substrate binding i.e. Asp216 and Thr334 were kept. Thus, same as in case of choline ion, Thr334 was found to be still involved in attractive interaction with the positively charged nitrogen atom of compound $\mathbf{5}$, but with remarkably lower interaction energy. On the contrary, the main stabilizing interaction for compound $\mathbf{5}$ was now provided by Asp216, the residue that is involved in the formation of a hydrogen bond with the inhibitor, as a result of methyl group substitution by a hydrogen atom in the ethanolamine group. The presence of a strong hydrogen bond created between Asp216 and compound $\mathbf{5}$ was observed in $88 \%$ of all structures explored during 50ns of MD simulations. Interestingly, the contribution of stabilizing van der Waals interactions between Met336 and inhibitor raised meaningfully compared to those created with choline ion. Finally, compound $\mathbf{5}$ was found to create a possible new contact with Ser503, but this interaction was not maintained along MD simulations and was observed in only $31 \%$ of all generated structures.

According to the results from CAVER3 Analyst, ${ }^{[48]}$ the volume of the active site must dramatically increase, from $\sim 145 \AA^{3}$ in choline bound conformer to $\sim 783 \AA^{3}$ in CutC in complex with compound $\mathbf{5}$, to provide sufficient space to bind a significantly larger molecule. However, such big pocket was not detected in the interior of the enzyme even in the case of 'choline-free' CutC $\left(\sim 262 \AA^{3}\right)$. Thus, the process of compound 5 binding in the active site of CutC must be coupled with large structural rearrangement of the enzyme. Hence, it would be expected that competitive binding of choline and compound $\mathbf{5}$ is initialized in an earlier phase 
i.e. in the recognition step, that should take place on the outer, more accessible part of the protein. This hypothesis was confirmed by blind docking studies, as described below.

The SwissDock ${ }^{[49,50]}$ server was used to identify a putative binding site of compound $\mathbf{5}$ in open conformer of CutC (see Supporting Information for details). The final location of the binding pockets for compound $\mathbf{5}$ in CutC was chosen based on the top score positions generated by SwissDock with highest FullFitness and estimated binding free energy.

Three top-scored clusters (cluster 1,2 and 3) characterized by very similar values of FullFitness (with difference smaller than 1 $\mathrm{kcal} \cdot \mathrm{mol}^{-1}$ ) indicated the existence of two possible binding pockets for compound $\mathbf{5}$ in open-CutC. As expected, none of the predicted binding pockets for this inhibitor was found in the active site, due to its relatively small size (as commented above). Therefore, binding pocket of cluster 1 and 2 is located in a very close surrounding of flexible $\alpha$-helix formed between 831 and 839 residues of $\mathrm{C}$-terminus. These clusters show different binding poses of compound $\mathbf{5}$, as presented in Figure 8 , indicating that a large size of the binding cavity and exposition to the water solvent permits reorientation of the inhibitor in its interior. Based on predicted values of energies, the rearrangement of the binding pose is expected to be energetically inexpensive. This was confirmed by MD simulations where very random contacts were observed between compound $\mathbf{5}$ and residues from this particular pocket (see Supporting Information for details). On the contrary, in cluster 3 , the binding pocket for compound $\mathbf{5}$ was localized in the interior of the protein and close to the entrance to the binding tunnel. In this binding mode two key interactions between Arg840 and Glu836 and compound 5 were identified. These were maintained during MD simulations as shown in Supporting Information.

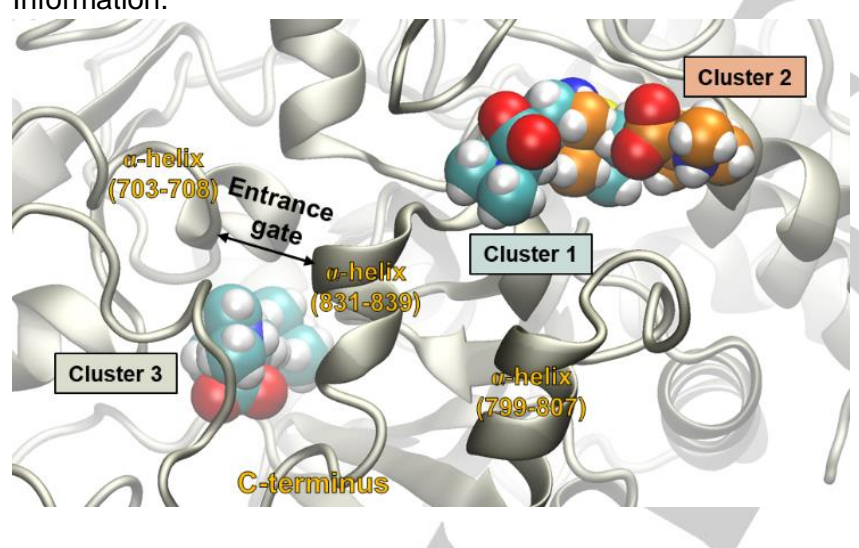

Figure 8. Predicted binding pockets for compound 5 defined by cluster 1,2 and 3 in the open-CutC protein conformer generated by SwissDock software.

Interestingly, a meaningful difference was observed for localization of binding pockets for choline ion in closed- and openCutC models (see Supporting Information). While in the closed conformer the most favorable binding site and the interactions between the ligand and the closest residues of the protein were identical to that observed in the X-ray structure, in the open-CutC the choline ion was bound distinctly far from the original position, closer to the entrance to the binding tunnel. The difference in location of binding pockets for substrate in both conformers confirms our previous conclusion that enzyme must undergo meaningful conformational changes before choline could reach the active site. Moreover, and what is crucial, it was found that the binding pocket predicted for compound 5 in open-CutC (in cluster 3) overlaps with the location of the cavity occupied by choline ion in the same protein conformer, although as expected compound 5 presents a distinctly different pattern of interactions (Supporting Information).

Thus, results from docking studies suggest that compound $\mathbf{5}$ is capable to block TMA production by two effects. First, the allosteric role of compound 5 that is manifested by binding close to flexible C-terminus that could result in a loss of its plasticity. However, the results from MD simulations studies did not provide any clue about specific interaction pattern between compound $\mathbf{5}$ and this particular binding pocket. Hence this scenario can be questionable. And second, the most probable, the inhibitor is directly involved in blocking the access of choline ion to the binding channel of CutC and, therefore, as experimentally observed decreasing the possibility of substrate binding. Finally, once compound 5 enters the active site of CutC, it is capable to establish several interactions that can favor its binding. In all, our computational results must be considered as a prediction since no experimental data about binding modes for compound $\mathbf{5}$ are available yet.

Variation in the reduction of TMA levels in different bacterial strains by small molecules targeting TMA-lyase has been reported. ${ }^{[17]}$ Therefore, the ability of compound $\mathbf{5}$ to impede the transformation of choline to TMA has been investigated in different bacterial strains, namely; Clostridium sporogenes, Proteus mirabilis, Klebsiella sp. MS 92-3, and E. coli MS 200-1. As shown in Figure 9, compound $\mathbf{5}$ was able to inhibit TMA production in all tested bacterial strains. However, the reduction in TMA levels from Proteus mirabilis was less pronounced in comparison to other bacterial strains. A similar attenuation in the ability to inhibit TMA production by CutC inhibitors in Proteus mirabilis has been previously demonstrated. ${ }^{[25]}$ Finally, we evaluated the ability of compound $\mathbf{5}$ to inhibit the conversion of choline to TMA in human fecal suspension in order to examine its efficacy in complex environment. Compund $\mathbf{5}$ demonstrated $\mathrm{EC}_{50}$ value of $29 \pm 3.4 \mu \mathrm{M}$ in the inhibition of TMA production from human fecal suspension in comparison to $\mathrm{EC}_{50}$ value of $88 \pm 9.2$ $\mu \mathrm{M}$ for 4 . These findings hold promise for compound $\mathbf{5}$ as a potential lead for in vivo studies and further optimization. 


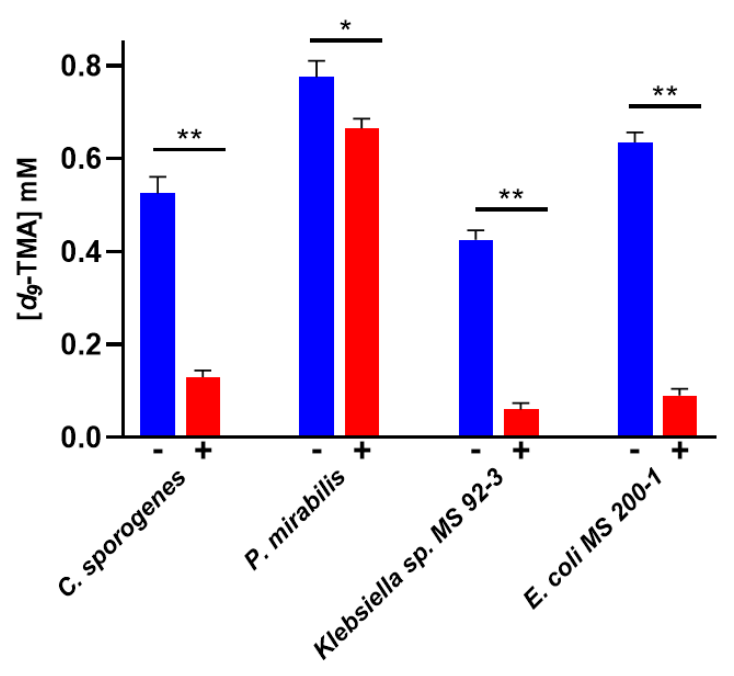

Figure 9. Levels of $d g$-TMA in Clostridium sporogenes, Proteus mirabilis, Klebsiella sp. MS 92-3, and E. coli MS 200-1 in the absence (-) and presence of $(+)$ compound $5(10 \mu \mathrm{M})$. Error bars represent standard deviation $(n=3)$. ( $p<0.05 ;{ }^{* \star} p<0.005$ relative to untreated control).

In summary, we have identified a histidine-based inhibitor, compound $\mathbf{5}$, that inhibits anaerobic metabolism of choline and consequently the production of TMA in whole cell assay. The new scaffold inhibits CutC with an $\mathrm{IC}_{50}$ value of $1.9 \pm 0.2 \mu \mathrm{M}$. Remarkably, compound $\mathbf{5}$ demonstrated broad spectrum CutC inhibition in various bacterial strains. Additionally, the efficacy of compound 5 to inhibit CutC in complex environment (e.g. human fecal suspension) was superior to previously reported CutC inhibitors. We have utilized blind docking studies in order to examine potential binding sites in CutC forcompound $\mathbf{5}$. These findings as well as metabolic stability of compound $\mathbf{5}$ would further enable the development of next generation of effective gut microbiota-targeted small molecules based on this scaffold as potential therapeutics for cardiovascular diseases and CKD.

\section{Experimental Section}

See the Supporting Information for experimental details and further biochemical data.

\section{Acknowledgements}

This work was supported by the Spanish Ministerio de Ciencia e Innovación (Grant PGC2018-094852-B-C21 and PID2019107098RJ-100), the Generalitat Valenciana (Grant SEJI/2020/007) and Universitat Jaume I (Grant UJI-A2019-04). KŚ thanks the MINECO for a Juan de la Cierva - Incorporación (ref. IJCl-2016-27503) contract. Authors acknowledge computational resources from the Servei d'Informàtica of Universitat Jaume I.

\section{Conflict of interest}

The authors declare no conflict of interest.

Keywords: Enzyme inhibitors $•$ choline $\cdot$ gut microbiota $•$ histidine $\cdot$ small molecules
[1] C. M. Guinane, P. D. Cotter, Therap. Adv. Gastroenterol. 2013, 6, 295308.

[2] Y. Wu, J. Wan, U. Choe, Q. Pham, N. W. Schoene, Q. He, B. Li, L. Yu, T. T. Y. Wang, Annu. Rev. Food Sci. T. 2019, 10, 389-408.

[3] R. L. Hughes, Front. Nutr. 2020, 6.

[4] F. Li, M. Wang, J. Wang, R. Li, Y. Zhang, Front. Cell. Infect. Microbiol. 2019, 9.

[5] W. H. W. Tang, T. Kitai, S. L. Hazen, Circ. Res. 2017, 120, 1183-1196.

[6] E. M. Onal, B. Afsar, A. Covic, N. D. Vaziri, M. Kanbay, Hypertens. Rese. 2019, 42, 123-140.

[7] D. Y. Li, W. H. W. Tang, Semin. Nephrol. 2018, 38, 193-205.

[8] J. M. Brown, S. L. Hazen, J. Biol. Chem. 2017, 292, 8560-8568.

[9] C. Missailidis, J. Hällqvist, A. R. Qureshi, P. Barany, O. Heimbürger, B. Lindholm, P. Stenvinkel, P. Bergman, PLoS One 2016, 11, e0141738.

[10] Z. Wang, E. Klipfell, B. J. Bennett, R. Koeth, B. S. Levison, B. Dugar, A E. Feldstein, E. B. Britt, X. Fu, Y. M. Chung, Y. Wu, P. Schauer, J. D. Smith, H. Allayee, W. H. Tang, J. A. DiDonato, A. J. Lusis, S. L. Hazen, Nature 2011, 472, 57-63.

[11] J. Miao, A. V. Ling, P. V. Manthena, M. E. Gearing, M. J. Graham, R. M. Crooke, K. J. Croce, R. M. Esquejo, C. B. Clish, D. Vicent, S. B. Biddinger, Nat. Commun. 2015, 6, 6498.

[12] M.-E. Dumas, R. H. Barton, A. Toye, O. Cloarec, C. Blancher, A Rothwell, J. Fearnside, R. Tatoud, V. Blanc, J. C. Lindon, S. C. Mitchell, E. Holmes, M. I. McCarthy, J. Scott, D. Gauguier, J. K. Nicholson, Proc Natl. Acad. Sci. USA 2006, 103, 12511.

[13] W. H. W. Tang, Z. Wang, D. J. Kennedy, Y. Wu, J. A. Buffa, B. AgatisaBoyle, X. S. Li, B. S. Levison, S. L. Hazen, Circ. Res. 2015, 116, 448455.

[14] A. B. Marcus, F. Gianfranco, M. E. Allan, Curr. Drug Metab. 2005, 6, 227-240.

[15] S. Craciun, E. P. Balskus, Proc. Natl. Acad. Sci. USA 2012, 109 21307-21312

[16] S. K. Krueger, D. E. Williams, Pharmacol. Ther. 2005, 106, 357-387.

[17] Z. Wang, A. B. Roberts, J. A. Buffa, B. S. Levison, W. Zhu, E. Org, X. Gu, Y. Huang, M. Zamanian-Daryoush, M. K. Culley, A. J. DiDonato, X. Fu, J. E. Hazen, D. Krajcik, J. A. DiDonato, A. J. Lusis, S. L. Hazen, Cell 2015, 163, 1585-1595

[18] A. B. Roberts, X. Gu, J. A. Buffa, A. G. Hurd, Z. Wang, W. Zhu, N. Gupta, S. M. Skye, D. B. Cody, B. S. Levison, W. T. Barrington, M. W. Russell, J. M. Reed, A. Duzan, J. M. Lang, X. Fu, L. Li, A. J. Myers, S. Rachakonda, J. A. DiDonato, J. M. Brown, V. Gogonea, A. J. Lusis, J. C. Garcia-Garcia, S. L. Hazen, Nat. Med. 2018, 24, 1407-1417.

[19] D. M. Shih, Z. Wang, R. Lee, Y. Meng, N. Che, S. Charugundla, H. Qi, J. Wu, C. Pan, J. M. Brown, T. Vallim, B. J. Bennett, M. Graham, S. L. Hazen, A. J. Lusis, J. Lipid Res. 2015, 56, 22-37.

[20] M. Warrier, D. M. Shih, A. C. Burrows, D. Ferguson, A. D. Gromovsky, A. L. Brown, S. Marshall, A. McDaniel, R. C. Schugar, Z. Wang, J. Sacks, X. Rong, T. A. Vallim, J. Chou, P. T. Ivanova, D. S. Myers, H. A. Brown, R. G. Lee, R. M. Crooke, M. J. Graham, X. Liu, P. Parini, P. Tontonoz, A. J. Lusis, S. L. Hazen, R. E. Temel, J. M. Brown, Cell Rep. 2015, 10 326-338.

[21] J. R. Cashman, K. Camp, S. S. Fakharzadeh, P. V. Fennessey, R. N. Hines, O. A. Mamer, S. C. Mitchell, G. P. Nguyen, D. Schlenk, R. L. Smith, S. S. Tjoa, D. E. Williams, S. Yannicelli, Curr. Drug Metab. 2003 4, 151-170.

[22] S. Craciun, J. A. Marks, E. P. Balskus, ACS Chem. Biol. 2014, 9, 1408 1413.

[23] S. Bodea, M. A. Funk, E. P. Balskus, C. L. Drennan, Cell Chem. Biol. 2016, 23, 1206-1216.

[24] M. Orman, S. Bodea, M. A. Funk, A. M.-d. Campo, M. Bollenbach, C. L. Drennan, E. P. Balskus, J. Am. Chem. Soc. 2019, 141, 33-37.

[25] M. Bollenbach, M. Ortega, M. Orman, C. L. Drennan, E. P. Balskus, ACS Med. Chem. Lett. 2020, DOI: https://doi.org/10.1021/acsmedchemlett.0c00005

[26] E. F. Enright, C. G. M. Gahan, S. A. Joyce, B. T. Griffin, Yale J. Biol. Med. 2016, 89, 375-382.

[27] M. Zimmermann, M. Zimmermann-Kogadeeva, R. Wegmann, A. L. Goodman, Nature 2019, 570, 462-467.

[28] M. B. Fisher, G. Labissiere, Curr. Drug Metab. 2007, 8, 694-699. 
[29] C. F. George, Clin. Pharmacokinet. 1981, 6, 259-274.

[30] L. Maier, M. Pruteanu, M. Kuhn, G. Zeller, A. Telzerow, E. E. Anderson, A. R. Brochado, K. C. Fernandez, H. Dose, H. Mori, K. R. Patil, P. Bork A. Typas, Nature 2018, 555, 623-628.

[31] A. Cornish-Bowden, Perspect. Sci. 2015, 4, 3-9.

[32] C. M. Breneman, K. B. Wiberg, J. Comput. Chem. 1990, 11, 361-373.

[33] Y. Zhao, D. G. Truhlar, Theor. Chem. Acc. 2008, 120, 215-241

[34] Y. Zhao, D. G. Truhlar, Acc. Chem. Res. 2008, 41, 157-167.

[35] A. Klamt, G. Schüürmann, J. Chem. Soc., Perkin Trans. 2 1993, 799805.

[36] J. Andzelm, C. Kölmel,A. Klamt, J. Chem. Phys.1995, 103, 9312-9320.

[37] V. Barone, M. Cossi, J. Phys. Chem. A 1998, 102, 1995-2001.

[38] M. Cossi, N. Rega, G. Scalmani, V. Barone, J. Comput. Chem. 2003, 24, 669-681.

[39] Y. Zhao, D. G. Truhlar, Theor. Chem. Acc. 2008, 120, 215-241.

[40] A. Waterhouse, M. Bertoni, S. Bienert, G. Studer, G. Tauriello, R. Gumienny, F. T. Heer, T. A. P. de Beer, C. Rempfer, L. Bordoli, R. Lepore, T. Schwede, Nucleic Acids Res. 2018, 46, W296-W303.

[41] S. Bienert, A. Waterhouse, T. A. P. de Beer, G. Tauriello, G. Studer, L. Bordoli, T. Schwede, Nucleic Acids Res. 2017, 45, D313-D319.

[42] N. Guex, M. C. Peitsch, T. Schwede, Electrophoresis 2009, 30, S162S173.

[43] G. Studer, C. Rempfer, A. M. Waterhouse, G. Gumienny, J. Haas, T. Schwede, Bioinformatics 2020, 36, 1765-1771.

[44] M. Bertoni, F. Kiefer, M. Biasini, L. Bordoli, T. Schwede, Sci. Rep. 2017, 7, 10480 .

[45] G. Kalnins, J. Kuka, S. Grinberga, M. Makrecka-Kuka, E. Liepinsh, M. Dambrova, K. Tars, J. Biol. Chem. 2015, 290, 21732-21740.

[46] Y. Duan, C. Wu, S. Chowdhury, M.C. Lee, G. Xiong, W.E.I. Zhang, R. Yang, P. Cieplak, R.A.Y. Luo, T. Lee, J. Caldwell, J. Wang, P. Kollman, J. Comput. Chem. 2003, 24, 1999-2012.

[47] J.C. Phillips, R. Braun, W. Wang, J. Gumbart, E. Tajkhorshid, E. Villa, C. Chipot, R.D. Skeel, L. Kalé, K. Schulten, J. Comput. Chem. 2005, 26, 1781-1802.

[48] A. Jurcik, D. Bednar, J. Byska, S. M. Marques, K. Furmanova, L. Daniel, P. Kokkonen, J. Brezovsky, O. Strnad, J. Stourac, A. Pavelka, M. Manak, J. Damborsky, B. Kozlikova, CAVER Analyst 2.0: Analysis and Visualization of Channels and Tunnels in Protein Structures and Molecular Dynamics Trajectories., Bioinformatics, bty386, 2018.

[49] A. Grosdidier, V. Zoete, O. Michielin, Nucleic Acids Res. 2011, 39(Web Server issue), W270-W277.

[50] A. Grosdidier, V. Zoete, O. Michielin, J. Comput. Chem. 2011, 32 , 2149-2159. 


\section{Entry for the Table of Contents}

Insert graphic for Table of Contents here.

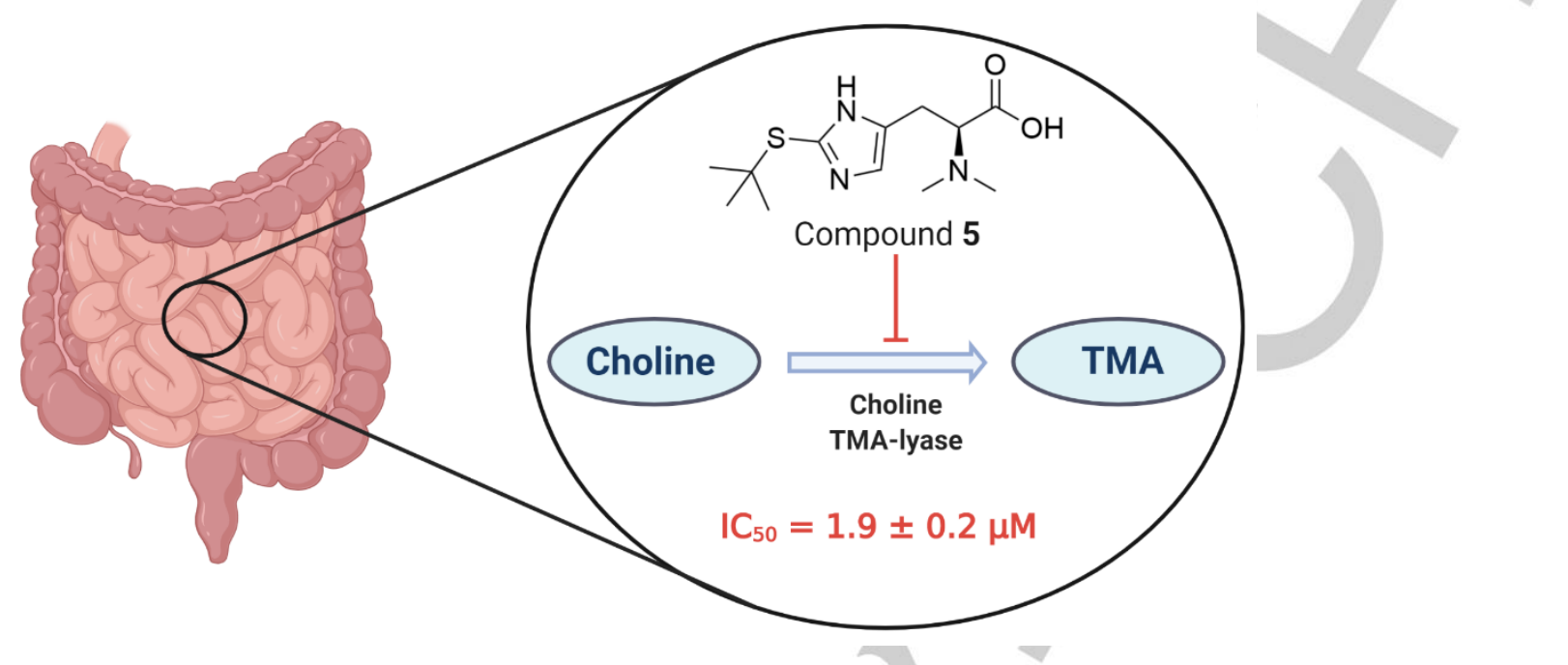

Inhibition of microbial choline trimethylamine(TMA)-lyase: We identified a novel scaffold that can inhibit trimethylamine (TMA) lyase expressed by human gut bacteria with an $\mathrm{IC}_{50}$ value of $1.9 \pm 0.2 \mu \mathrm{M}$. The reported inhibitor displayed universal inhibition of TMA lyase in multiple bacterial strains and maintained the inhibitory activity in complex biological environment. 